\title{
Evaluating the effect of lane width and roadside configurations on speed, lateral position and likelihood of comfortable overtaking in exclusive motorcycle lane
}

\begin{abstract}
Construction of exclusive motorcycle lanes is one of the measures to reduce motorcycle fatalities. Previous studies highlighted the risk of crashes with roadside objects and the tendency of motorcyclists to ride with excessive speed on exclusive motorcycle lanes. However, the risk of same-direction crashes on exclusive motorcycle lanes was not explored in much detail, especially on the impact of lane geometry and roadside configurations. This study used naturalistic riding data to determine the effects of lane width and roadside configurations on overtaking speed, lateral position and likelihood of comfortable overtaking on tangential sections of an exclusive motorcycle lane. Twenty-nine recruited motorcyclists rode the instrumented motorcycles along a $20 \mathrm{~km}$ stretch of an exclusive motorcycle lane along a major urban road. Results revealed that both the roadside configurations and lane width significantly affect the participants' lateral position, while the roadside configurations only affects the overtaking speed. Participants' overtaking speeds and the front motorcycles' lateral position contribute significantly to the likelihood of comfortable overtaking in exclusive motorcycle lanes. The findings highlight the importance of micro-level behavior indicators in improving the design and overall safety of the exclusive motorcycle facility.
\end{abstract}

Keyword: Comfortable overtaking; Instrumented motorcycle; Lane geometry; Motorcycle facility; Naturalistic riding data; Roadside configurations 